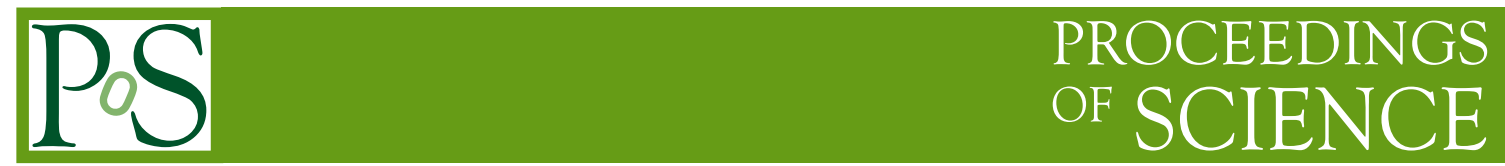

\title{
Charged Higgs search with SuperB
}

\author{
Alberto Lusiani*i \\ Scuola Normale Superiore and INFN, Pisa \\ E-mail: alberto.lusianiepi.infn.it
}

The SuperB project aims at accumulating $75 \mathrm{ab}^{-1}$ of $e^{+} e^{-}$collisions at the $\Upsilon(4 S)$ over a period of five years. By improving the experimental precision of $B$ meson branching fractions that have only suppressed Standard Model amplitudes, Super $B$ can significantly improve the present sensitivity to the presence of the charged Higgs boson included in several New Physics models.

Prospects for Charged Higgs Discovery at Colliders

October 8-11, 2012

Uppsala University Sweden

\footnotetext{
*Speaker.

${ }^{\dagger}$ Representing the Super $B$ collaboration
} 


\section{Introduction}

The Super $B$ project aims at accumulating $75 \mathrm{ab}^{-1}$ of $e^{+} e^{-}$collisions at the $\Upsilon(4 S)$ over a period of five years. The study of a large number of $B$ meson decays in an especially clean environment provides an effective tool to search for the charged Higgs boson.

Super $B$ can search for the charged Higgs by looking at deviations with respect to the Standard Model predictions in the processes $B \rightarrow \tau \nu, B \rightarrow \mu \nu, B \rightarrow D^{(*)} \ell v, B \rightarrow s \gamma, B \rightarrow s \ell^{+} \ell^{-}$, and by the measurement of $C P V$ in tau decay. A subset of such channels were studied and in the following the respective sensitivities for the charged Higgs are presented.

\section{2. $B \rightarrow \tau \nu$}

Within the Standard Model (SM) the charged $B$ meson can decay through a virtual $W$ to a charged lepton and its corresponding neutrino and the branching ratio for the process is given by

$$
\mathscr{B}(B \rightarrow \ell v)=\frac{G_{F}^{2} m_{B}}{8 \pi} m_{\ell}^{2}\left(1-\frac{m_{\ell}^{2}}{m_{B}^{2}}\right) f_{B}^{2}\left|V_{u b}\right|^{2} \tau_{B}
$$

where $m_{B}$ and $m_{l}$ are the masses of the $B$ and of the lepton, $\tau_{B}$ is the $B$ lifetime, $G_{F}$ is the Fermi constant, $V_{u b}$ is the CKM element and $f_{B}$ is the $B$ meson decay constant.

The precision of the SM is limited by $m_{B}, V_{u b}$ and $f_{B}$. Using lattice QCD calculations and experimental data elaborated with UTFIT [1], a recent SM prediction is $\mathscr{B}(B \rightarrow \tau v)=(0.82 \pm$ $0.08) \cdot 10^{-4}$ [2]. This is to be compared with the most recent average of experimental measurements $\mathscr{B}(B \rightarrow \tau v)=(1.14 \pm 0.23) \cdot 10^{-4}[3]$. Muon and electron branching fractions are much smaller, because of the helicity suppression represented by the $m_{l}^{2}$ factor.

A charged Higgs boson exchange interferes with the charged weak coupling and modifies the SM predicted amplitude. A minimal model with an additional Higgs doublet (type-II 2HDM [4]) predicts:

$$
\mathscr{B}(B \rightarrow \ell v)=\frac{G_{F}^{2} m_{B}}{8 \pi} m_{\ell}^{2}\left(1-\frac{m_{\ell}^{2}}{m_{B}^{2}}\right) f_{B}^{2}\left|V_{u b}\right|^{2} \tau_{B} \times\left(1-\tan ^{2} \beta \frac{m_{B}^{2}}{m_{H}^{2}}\right)^{2}
$$

where $m_{H}$ is the charged Higgs mass and $\tan \beta$ is the ratio of the Higgs vacuum expectation values. Minimal Supersymmetry (MSSM) predicts [5]:

$$
\mathscr{B}(B \rightarrow \ell v)=\frac{G_{F}^{2} m_{B}}{8 \pi} m_{\ell}^{2}\left(1-\frac{m_{\ell}^{2}}{m_{B}^{2}}\right) f_{B}^{2}\left|V_{u b}\right|^{2} \tau_{B} \times\left(1-\frac{\tan ^{2} \beta}{1+\bar{\varepsilon}_{0} \tan \beta} \frac{m_{B}^{2}}{m_{H}^{2}}\right)^{2}
$$

where $\bar{\varepsilon}_{0}$ is a SUSY correction factor

We compare the present and future SuperB experimental sensitivity to the type-II 2HDM Higgs by assuming that we measure exactly the SM prediction in both cases. In this case, upper limits can be set on the New Physics (NP) amplitude parameters in the $\tan \beta-m_{H}$ plane, according to the uncertainties on the measurement and the SM prediction. When assuming that we measure today the SM prediction, we keep the relative uncertainty constant. Regarding the SuperB experimental uncertainty, we extrapolate the relative uncertainty of the recent $B A B A R$ hadronic recoil tag analysis $\left[(1.83 \pm 0.56) \cdot 10^{-4}[6]\right]$ from the $468 \mathrm{fb}^{-1}$ of the $B A B A R$ sample to the expected $75 \mathrm{ab}^{-1}$ of Super $B$ : 


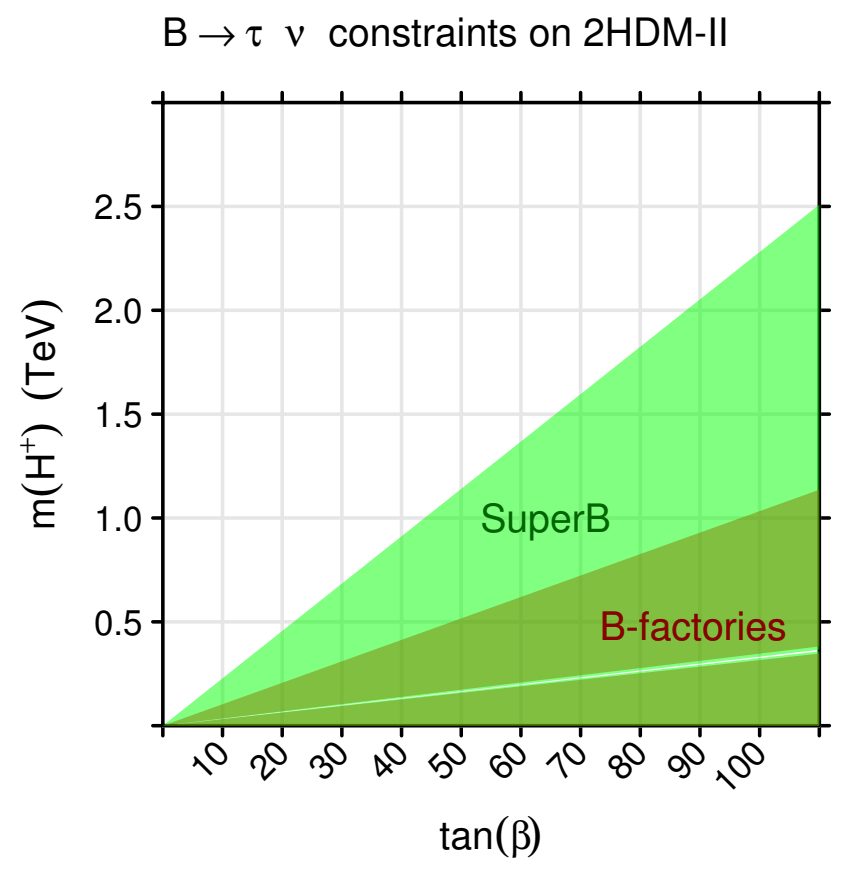

Figure 1: Present ( $B$-factories) and future (Super $B$ ) 90\% CL exclusion area for the charged Higgs boson of the type-II 2HDM model, assuming that exactly the SM prediction is measured in both cases for the $B \rightarrow \tau \nu$ branching fraction.

this correspond to $\approx 2.4 \%$ if we assume that also systematic contributions can be reduced proportionally to the square root of the integrated luminosity. To account for irreducible systematics, we scale up the expected SuperB measurement uncertainty to $3.5 \%$. We also expect that the uncertainty of the SM prediction can be reduced from the present $\approx 10 \%$ to $3.5 \%$ by improvements in both lattice QCD calculations and experimental measurements. Figure 1 reports the corresponding excluded area at $90 \% \mathrm{CL}$ for the type-II $2 \mathrm{HDM}$ model in the $\tan \beta-m_{H}$ plane obtained today with the $B$-factories BABAR and Belle and expected for SuperB. Since the NP amplitude has opposite sign to the SM amplitude, this measurement cannot exclude a small area corresponding to the combination of model parameters that make the NP amplitude equal to twice the SM amplitude within uncertainties.

\section{3. $B \rightarrow s \gamma$}

With respect to $B \rightarrow \tau \nu$, the inclusive $B \rightarrow s \gamma$ branching fraction $^{1}$ is more difficult to predict in the SM. Recent NNLO calculations give the SM prediction $\mathscr{B}(B \rightarrow s \gamma)_{\mathrm{SM}}=(3.15 \pm 0.23)$. $10^{-4}[7,8]$. This corresponds to a $7.3 \%$ relative uncertainty. There is no general consensus on how much the SM prediction uncertainty can be reduced in the next decade, although a relatively short term improvement to 5\% appears to be quite possible [9]. On the experimental side, the present

\footnotetext{
${ }^{1}$ Here and in the following $E_{\gamma}>1.6 \mathrm{GeV}$ is assumed.
} 


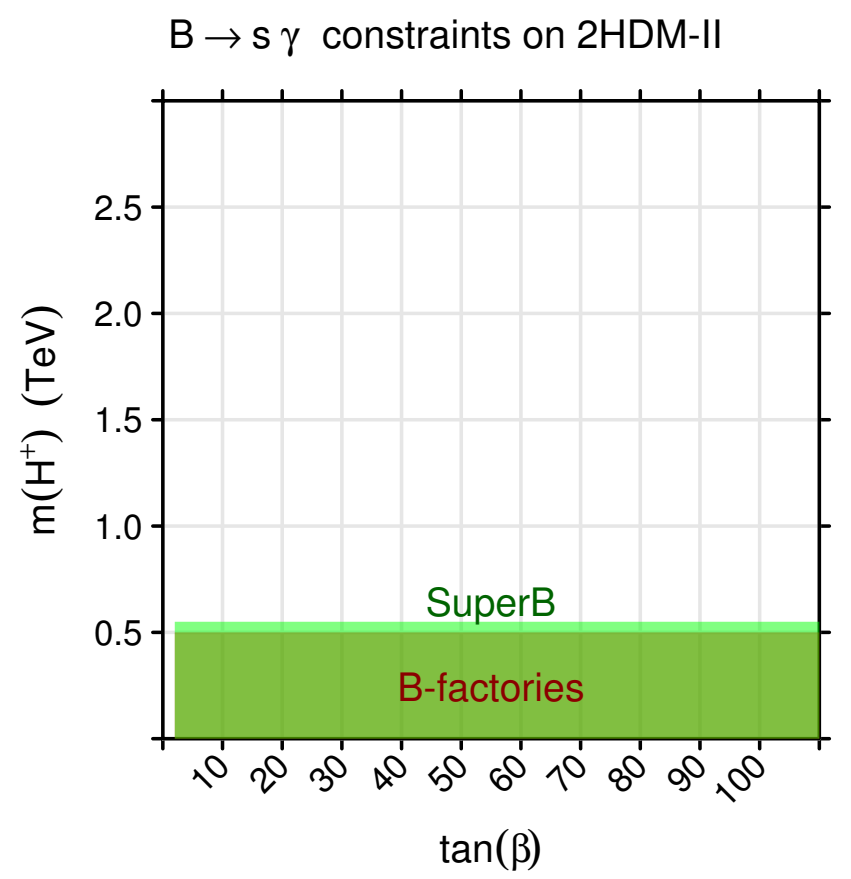

Figure 2: Present ( $B$-factories) and future (Super $B$ ) $90 \%$ CL exclusion area for the charged Higgs boson mass of the type-II $2 \mathrm{HDM}$ model, assuming that exactly the SM prediction is measured in both cases for the $B \rightarrow s \gamma$ branching fraction with $E_{\gamma}>1.6 \mathrm{GeV}$.

accuracy is $6.5 \%$ (from $\mathscr{B}(B \rightarrow s \gamma)_{\mathrm{EXP}}=343 \pm 22$, world average in the August 2012 web update of the early 2012 HFAG report [10]).

The effects of the presence of charged Higgs amplitudes from the type-II 2HDM model have been evaluated at NNLO [11]. The level of agreement between theory and experiment set a lower limit for the mass of the charged Higgs boson at $m_{H}>360 \mathrm{GeV}$ at $95 \% \mathrm{CL}$.

We expect that SuperB can reduce the experimental uncertainty from $6.5 \%$ to $3 \%$ exploiting the larger statistics with the cleanest measurement of $B \rightarrow s \gamma$, based on fully reconstructed hadronic $B$ decay tags. In order to compare the situation today with the Super $B$ expectations, we assume that in both cases exactly the SM prediction is measured. In this case, the lower limit on the charged Higgs mass at $90 \% \mathrm{CL}$ is expected to improve from about $500 \mathrm{GeV}$ to about $550 \mathrm{GeV}$, using the plots provided in Ref. [11]. Figure 2 reports the estimated limits at 90\% CL on the charged Higgs mass today and with SuperB, for the type-II 2HDM model. The vertical scale is the same as for Figure 1 to allow a direct comparison.

\section{4. $B \rightarrow D^{(*)} \tau \nu$ and other channels}

NP models with charged Higgs bosons affect the amplitude of the $B \rightarrow D \tau \nu$ and $B \rightarrow D^{*} \tau \nu$ processes in a similar way as $B \rightarrow \tau \nu$, with an opposite-sign amplitude due to the Higgs exchange. In particular, the statistical power of the $B \rightarrow D \tau v$ measurements exceeds the other channel and, although it is less powerful than $B \rightarrow \tau \nu$, it is maximally sensitive approximately in the small 
area in the $\tan \beta-m_{H}$ plane where $B \rightarrow \tau \nu$ loses sensitivity. To reduce uncertainties from QCD corrections, it is convenient to compare the experiment with theory on the ratio $\mathscr{R}=\mathscr{B}(B \rightarrow$ $D \tau v) / \mathscr{B}(B \rightarrow D e v)$, whose SM prediction has a relative precision around 5\% [12]. The experimental value has been recently measured to be $\mathscr{B}(B \rightarrow D \tau \nu)=0.440 \pm 0.058 \pm 0.042$ [13]. There is no present estimate for the Super $B$ precision on this channel, however this measurement is less sensitive than $B \rightarrow \tau \nu$.

Super $B$ will be sensitive to NP models containing a charged Higgs in several additional processes, including $B \rightarrow \mu v, B \rightarrow s l^{+} l^{-}$, and $C P$-violating $\tau$ decays. Precise estimates on the charged Higgs sensitivity are not available but are expected to be weaker than $B \rightarrow \tau v$, with the possible exception of $B \rightarrow \mu v$.

\section{Conclusion}

Super $B$ measurements provide several handles sensitive to the presence of the charged Higgs boson predicted by several NP models. $B \rightarrow \tau \nu$ is the most powerful measurement for charged Higgs sensitivity, which reaches masses even beyond $2 \mathrm{TeV}$ for high $\tan \beta$ values for simple models like the type-II $2 \mathrm{HDM}$.

\section{References}

[1] UTfit Collaboration, M. Bona et al., An Improved Standard Model Prediction of $B \rightarrow \tau v$ and its Implications for New Physics, Phys.Lett. B687 (2010) 61-69, [arXiv: 0908 . 3470 ].

[2] C. Tarantino, Flavor Lattice QCD in the Precision Era, arXiv: 1210.0474.

[3] S. Fajfer and I. Nisandzic, Theory of $B \rightarrow \tau v$ and $B \rightarrow D^{(*)} \tau \nu$, arXiv:1301.1167.

[4] W.-S. Hou, Enhanced charged Higgs boson effects in B- $\rightarrow$ tau anti- neutrino, mu anti-neutrino and $b$ $\rightarrow$ tau anti-neutrino + X, Phys. Rev. D48 (1993) 2342-2344.

[5] A. Akeroyd and S. Recksiegel, The Effect of $H^{ \pm}$on $B^{ \pm} \rightarrow \tau^{ \pm} v_{\tau}$ and $B^{ \pm} \rightarrow \mu^{ \pm} v_{\mu}$, J.Phys. G29 (2003) 2311-2317, [hep-ph/0306037].

[6] BaBar Collaboration, J. Lees et al., Evidence of $B \rightarrow \tau v$ decays with hadronic $B$ tags, arXiv: 1207.0698.

[7] M. Misiak and M. Steinhauser, NNLO QCD corrections to the anti-B $\rightarrow X /$ s gamma matrix elements using interpolation in $m(c)$, Nucl. Phys. B764 (2007) 62-82, [hep-ph/ 0609241$].$

[8] M. Misiak et al., The first estimate of $B($ anti-B $->X / s$ gamma $)$ at $O($ alpha(s)**2), Phys. Rev. Lett. 98 (2007) 022002, [hep-ph/0609232].

[9] A. Crivellin, 2012. private communication.

[10] Heavy Flavor Averaging Group Collaboration, Y. Amhis et al., Averages of b-hadron, c-hadron, and tau-lepton properties as of early 2012, arXiv:1207.1158.

[11] T. Hermann, M. Misiak, and M. Steinhauser, $\bar{B} \rightarrow X_{s} \gamma$ in the Two Higgs Doublet Model up to Next-to-Next-to-Leading Order in QCD, JHEP 1211 (2012) 036, [arXiv: 1208 . 2788].

[12] S. Fajfer, J. F. Kamenik, and I. Nisandzic, On the $B \rightarrow D^{*} \tau \bar{v}_{\tau}$ Sensitivity to New Physics, Phys.Rev. D85 (2012) 094025, [arXiv: 1203.2654 ]. 
[13] BaBar Collaboration Collaboration, J. Lees et al., Evidence for an excess of $\bar{B} \rightarrow D^{(*)} \tau^{-} \bar{v}_{\tau}$ decays, Phys.Rev.Lett. 109 (2012) 101802, [arXiv: 1205.5442$].$ 